\title{
FADIGA ISOTÉRMICA E TERMOMECÂNICA DE UMA SUPERLIGA À BASE DE NIQUEL
}

Carlos Carvalho Engler-Pinto Júnior ' Dirceu Spinelli ${ }^{2}$

\section{Resumo}

Os gradientes térmicos originados durante regimes transientes em operações de liga/desliga produzem complexos carregamentos mecânicos e térmicos de fadiga, que limitam a vida de palhetas de turbina e de outros componentes de motores que operam a altas temperaturas. Assim, torna-se imprescindível uma avaliação mais criteriosa e confível da fadiga não isotérmica. Este trabalho investiga o comportamento em fadiga isotérmica de baixo ciclo (FBC) e fadiga termomecânica (FTM) de uma superliga à base de Ni, CM 247LC-DS, extensivamente utilizada a altas temperaturas. Os ensaios mecânicos foram realizados em temperaturas entre $600^{\circ} \mathrm{C}$ e $1.000^{\circ} \mathrm{C}$. O comportamento mecânico da liga foi fortemente afetado no intervalo $800^{\circ} \mathrm{C}-1.000^{\circ} \mathrm{C}$ e o comportamento tensão-deformação cíclico observado para todo o intervalo de temperatura obedeceu à equação de Ramberg-Osgood. Um modelo simplificado de tensão-deformação não isotérmico, baseado no conceito de plasticidade linear foi proposto e descreveu o comportamento de fadiga termomecânica observado, tanto para ciclos em fase, como para fora de fase.

Palavras-chave: Superliga; Fadiga isotérmica; Fadiga termomecânica.

\section{ISOTHERMAL AND THERMOMECHANICAL FATIGUE OF A NICKEL-BASE SUPERALLOY}

\begin{abstract}
Thermal gradients arising during transient regimes of start-up and shutdown operations produce a complex thermal and mechanical fatigue loading which limits the life of turbine blades and other engine components operating at high temperatures. More accurate and reliable assessment under non-isothermal fatigue becomes therefore mandatory. This paper investigates the nickel base superalloy CM 247LC-DS under isothermal low cycle fatigue (LCF) and thermomechanical fatigue (TMF). Test temperatures range from $600^{\circ} \mathrm{C}$ to $1,000^{\circ} \mathrm{C}$. The behavior of the alloy is strongly affected by the temperature variation, especially in the $800^{\circ} \mathrm{C}-1,000^{\circ} \mathrm{C}$ range. The Ramberg-Osgood equation fits very well the observed isothermal behavior for the whole temperature range. The simplified non-isothermal stress-strain model based on linear plasticity proposed to represent the thermo-mechanical fatigue behavior was able to reproduce the observed behavior for both in-phase and out-of-phase TMF cycling.
\end{abstract}

Keywords: Superalloy; Isothermal fatigue; Thermomechanical fatigue.

\section{INTRODUÇÃO}

As superligas à base de $\mathrm{Ni}$ obtidas por solidificação direcional (SD) são materiais utilizados na fabricação de palhetas de turbinas a gás estacionárias. $A$ introdução do processo de solidificação direcional (SD) e de obtenção de monocristais (MC) destas ligas levou a melhores propriedades de resistência à fluência e oxidação, melhor desempenho a altas temperaturas e, consequentemente, a maior durabilidade das palhetas $[1,2]$.

Nos processos de obtenção SD e MC, a direção cristalográfica [00I] é orientada paralelamente ao eixo principal da palheta. Esta direção apresenta módulo de elasticidade mais baixo, e assim gera tensões térmicas menores durante as operações de resfriamento e aquecimento do componente. Os fatores limitantes para a vida destas palhetas são: fadiga termomecânica, fluência e oxidação no aerofólio, além de fadiga de baixo ciclo a altas temperaturas na seção da raiz da palheta [3]. Durante os regimes transientes de operações liga/desliga, as palhetas são submetidas à fadiga termomecânica devido aos gradientes gerados pelas variações de temperatura durante o resfriamento. Uma descrição correta da resposta tensão-deformação cíclica a altas temperaturas

\footnotetext{
'Engenheiro Mecânico, Técnico Especialista, Ford Motor Company, Material Science Department, Dearborn, Michigan, EUA. E-mail: cenglerp@ford.com ${ }^{2}$ Engenheiro Mecânico, Professor Sênior, Departamento de Engenharia de Materiais, Escola de Engenharia de São Carlos, Universidade de São Paulo - USP, São Carlos, SP, Brasil. E-mail: dspinell@sc.usp.br
} 
constitui um dos pré-requisitos para qualquer modelamento de durabilidade a altas temperaturas [4].

Os objetivos principais desta investigação são o estudo do comportamento cíclico isotérmico e termomecânico da superliga CM247LC-DS, obtida pelo processo de solidificação direcional, no intervalo de temperaturas entre $600^{\circ} \mathrm{C}$ e $1.000^{\circ} \mathrm{C}$ e o desenvolvimento de um modelo para a previsão do comportamento tensão-deformação cíclico, no mesmo intervalo de temperatura.

\section{MATERIAIS E MÉTODOS}

A superliga à base de níquel denominada de CM247LC-DS, cuja composição química é mostrada na Tabela I, é derivada da composição básica da superliga MAR-M247. Os principais benefícios obtidos com a mudança na composição química da superliga original foram: a) redução da porcentagem de carbono de aproximadamente para a metade, melhorando assim a microestrutura de carbonetos, estabilidade e ductilidade; b) redução dos elementos $\mathrm{Zr}$ e Ti, produzindo assim, um aumento da resistência ao trincamento dos contornos dos grãos colunares, sem, contudo, afetar os parâmetros de resistência mecânica da superliga; c) diminuição dos níveis dos elementos $\mathrm{W}$ e Mo, com a finalidade de minimizar a formação de plaquetas dos carbonetos do tipo $\mathrm{M}_{6} \mathrm{C}$, fase $\mu$ ou plaquetas $\alpha$ ou agulhas $\Omega$ [4].

A superliga foi submetida a tratamentos térmicos multipasses, que consta de um tratamento de solubilização a $1.260^{\circ} \mathrm{C}$ por $2 \mathrm{~h}$, seguido de dois tratamentos de precipitação, um a $1.080^{\circ} \mathrm{C}$ por $4 \mathrm{~h}$ e outro a $870^{\circ} \mathrm{C}$ por $20 \mathrm{~h}$.

Os ensaios de fadiga isotérmica foram realizados em corpos de prova sólidos convencionais de acordo com a norma ASTM E 606-04 [5], enquanto que os ensaios de fadiga termomecânica foram realizados em corpos de prova tubulares, de acordo com a norma ASTM E 2368-04 [6]. A partir de placas fundidas, os corpos de prova foram fabricados pelo processo de eletro-erosão, com a direção longitudinal paralela aos grãos colunares. A superfície externa foi torneada e polida mecanicamente com pasta de diamante de I $\mu \mathrm{m}$. A superfície do furo dos corpos de prova para os ensaios de fadiga termomecânica foi polido com uma ferramenta especialmente projetada, com a finalidade de se obter um acabamento superficial interno com rugosidade comparável à da superfície externa.

Todos os ensaios foram realizados em uma máquina de ensaios servo-hidráulica de circuito fechado, com capacidade de $100 \mathrm{kN}$, especialmente equipada para realização de ensaios de fadiga termomecânica, sob controle de deformação total.
Os corpos de prova foram aquecidos por meio de um conversor de rádio frequência e um pirômetro ótico foi usado para monitorar a temperatura do ensaio. Um fluxo de argônio de vazão variável foi aplicado através do orifício existente na garra de fixação dos corpos de prova tubulares, com o intuito de controlar o gradiente térmico através da parede do tubo, bem como diminuir o processo de oxidação da superfície interna. Os experimentos foram monitorados por um microcomputador conectado a um sistema de controle e aquisição de dados usando um programa desenvolvido em ambiente HP VEE-Test. Foi permitido um máximo de $10^{\circ} \mathrm{C} / \mathrm{s}$, tanto para a taxa de aquecimento, como para a taxa de resfriamento. Este procedimento produziu um gradiente térmico menor que $10^{\circ} \mathrm{C}$ ao longo do comprimento de medida do corpo de prova. A Figura I ilustra os ciclos de fadiga termomecânica aplicados, em-fase (Figura Ia), fora-de-fase (Figura Ib) e bi-térmico (Figura Ic) que segue um padrão quadrado.

\section{RESULTADOS E DISCUSSÕES}

Para a obtenção das curvas tensão-deformação cíclicas foi empregado o método de ensaio incremental de múltiplos passos [7]. Esta metodologia foi aplicada tanto para os ensaios de fadiga isotérmica de baixo ciclo, como para os de fadiga termomecânica.

A liga CM 247LC-DS mostra uma diferença marcante no comportamento em fadiga isotérmica de baixo ciclo em temperaturas maiores que $800^{\circ} \mathrm{C}$. Esta diferença de comportamento pode vista na Figura 2, que compara as curvas de histerese obtidas nas temperaturas de $600^{\circ} \mathrm{C}, 900^{\circ} \mathrm{C}$ e $1.000^{\circ} \mathrm{C}$, para intervalos similares de deformação.

A histerese a $600^{\circ} \mathrm{C}$ é muito similar àquelas obtidas às temperaturas de $700^{\circ} \mathrm{C}$ e $800^{\circ} \mathrm{C}$, respectivamente, e são caracterizadas por altos limites de escoamento cíclicos, combinadas com expoentes de encruamento relativamente baixos. Ou seja, o material flui facilmente uma vez
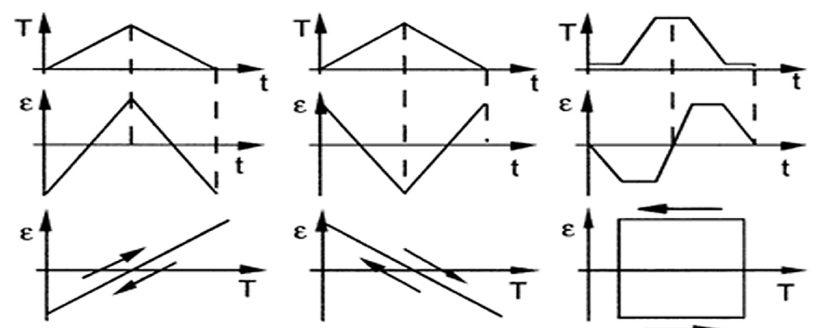

a) Em fase (EF)

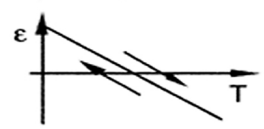

b) Fora de fase (FF)

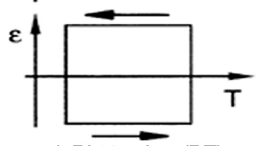

c) Bi-térmico (BT)

Figura I. Ciclos de fadiga termodinâmica aplicados, (a) em fase; (b) fora de fase; e (c) bi-térmico.

Tabela I. Composição química da liga CM247LC-DS (\% em peso)

\begin{tabular}{cccccccccc}
\hline $\mathbf{C}$ & Al & Co & Cr & Mo & Fe & Ta & Ti & W & Hf \\
\hline 0,073 & 5,63 & 9,3 & 8,1 & 0,50 & 0,022 & 3,19 & 0,70 & 9,5 & 1,4 \\
\hline
\end{tabular}




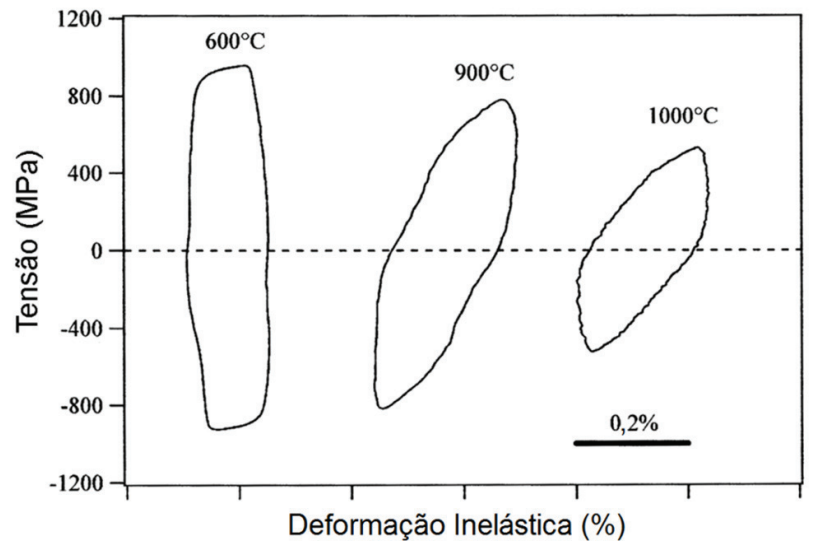

Figura 2. Curvas de histerese isotérmicas para diferentes temperaturas.

alcançado o limite de escoamento. A $900^{\circ} \mathrm{C}$ e $1.000^{\circ} \mathrm{C}$, entretanto, há uma diminuição significativa nos limites de escoamento cíclicos, enquanto que os expoentes de encruamento tendem a serem maiores. Este comportamento pode ser visto na Figura 3, onde estão sumarizados todos os resultados dos ensaios cíclicos isotérmicos da variação de tensão em função da variação de deformação inelástica para os ciclos de histerese estabilizados, em duas diferentes taxas de deformação mecânica, $10^{-3} \mathrm{~s}^{-1} \mathrm{e}$ $2 \times 10^{-4} \mathrm{~s}^{-1} \mathrm{e}$ em várias temperaturas.

Para as duas condições investigadas, a liga não se mostrou sensível às taxas de deformação aplicadas, para temperaturas de $600^{\circ} \mathrm{C}$ até $800^{\circ} \mathrm{C}$. Entretanto, para as temperaturas de $900^{\circ} \mathrm{C}$ e $1.000^{\circ} \mathrm{C}$ pode ser observado que a variação da deformação inelástica aumenta e a variação da tensão diminui, com a redução da taxa de deformação. Isto indica que, nestas temperaturas mais elevadas, os mecanismos de fluência por difusão, especificamente, operam mais efetivamente.

As curvas tensão-deformação cíclicas termomecânicas foram geradas apenas para a taxa de deformação de $2 \times 10^{-4} \mathrm{~s}^{-1}$ Os resultados obtidos estão mostrados na Figura 4, juntamente com os resultados isotérmicos obtidos para a mesma taxa de deformação. O comportamento em fadiga termomecânica, para os carregamentos em fase e fora de fase mostra-se similar e os pontos obtidos situam-se entre as curvas obtidas para os ensaios a $600^{\circ} \mathrm{C}-800^{\circ} \mathrm{C}$ e para $1.000^{\circ} \mathrm{C}$. Entretanto, os resultados do ciclo de FTM bi-térmico seguem a mesma tendência que os dos ciclos isotérmicos a $1.000^{\circ} \mathrm{C}$. Deve ser lembrado que o ciclo de carregamento bi-térmico é caracterizado pela manutenção da deformação enquanto a temperatura varia e a deformação mecânica é cíclica a temperaturas constantes, de $600^{\circ} \mathrm{C}$ e $1.000^{\circ} \mathrm{C}$, neste caso.

O comportamento cíclico de tensão-deformação é geralmente representado pela equação de Ramberg- Osgood, que é uma lei de potência dada pela Equação I:

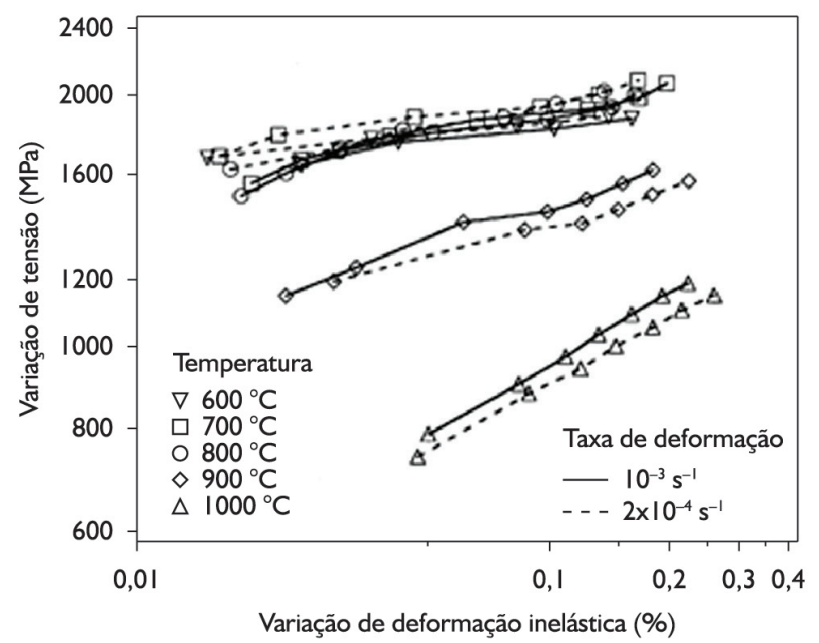

Figura 3. Curvas isotérmicas de tensão-deformação cíclicas.

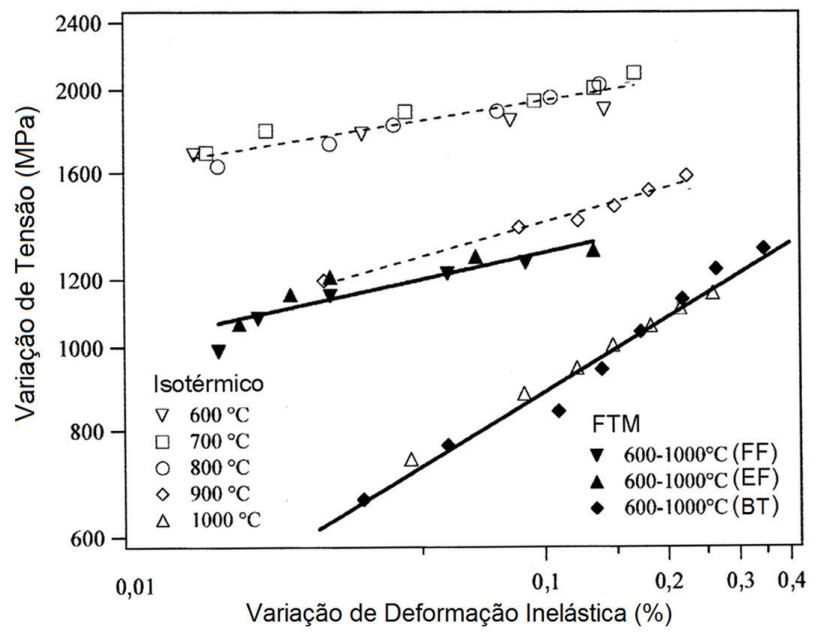

Figura 4. Curvas de tensão-deformação cíclicas isotérmicas e termomecânicas.

$$
\frac{\Delta \sigma}{2}=K^{\prime}\left(\frac{\Delta \varepsilon_{\text {in }}}{2}\right)^{n^{\prime}}
$$

Onde $\Delta \sigma$ é a variação de tensão, $\Delta \varepsilon_{\text {in }}$ é a variação de deformação inelástica, $K$ ' é o coeficiente de resistência cíclico e $n$ ' é o expoente de encruamento cíclico.

Para o modelamento do comportamento cíclico da superliga CM 247LC-DS, uma representação constitutiva com comportamento elástico-linear-plástico foi proposta, como esquematizada na Figura 5.

Supõe-se que a deformação inelástica ocorra linearmente, seguindo as duas superfícies definidas pelas Equações 2 e 3 , quando o material é deformado em tração e compressão, respectivamente:

$$
\begin{aligned}
& \sigma=\mathrm{S}_{\mathrm{e}}+\mathrm{E}_{\mathrm{p}} \cdot \varepsilon_{\text {in }} \\
& \sigma=-\mathrm{S}_{\mathrm{e}}+\mathrm{E}_{\mathrm{p}} \cdot \varepsilon_{\text {in }}
\end{aligned}
$$


Onde $\sigma$ é a tensão aplicada, $S_{e}$ é o limite de escoamento cíclico, $E_{p}$ é a inclinação da reta plástica e $\varepsilon_{\text {in }}$ é a deformação inelástica.

Para as tensões entre as superfícies definidas pelas Equações 2 e 3, o modelo assume que o material comporta-se elasticamente de acordo com a Equação 4.

$$
\sigma=\mathrm{E} \cdot\left(\varepsilon_{\mathrm{mec}}-\varepsilon_{\text {in }}\right)
$$

Onde $E$ é o módulo de elasticidade, $\varepsilon_{\text {mec }}$ é a deformação mecânica e $\varepsilon_{\text {in }}$ é a deformação inelástica.

Para a determinação das constantes do modelo elástico-linear-plástico, foi assumido que a quantidade de trabalho plástico $W_{p}=\int \sigma d \varepsilon_{\text {in }}$ medido sob a curva tensão-deformação inelástica, fosse a mesma que para uma dada deformação de referência $\varepsilon_{\text {in }}^{\text {ref }}$. Neste caso, as constantes $S_{e}$ e $E_{p}$ podem ser determinadas por meio das Equações 5 e 6 .

$$
\begin{aligned}
& S_{e}=K^{\prime} \cdot\left(\varepsilon_{i n}^{\text {ref }}\right)^{n^{\prime}} \cdot\left(\frac{I-n^{\prime}}{I+n^{\prime}}\right) \\
& E_{p}=K^{\prime} \cdot\left(\varepsilon_{\text {in }}^{\text {ref }}\right)^{n^{\prime}-1} \cdot\left(\frac{2 n^{\prime}}{I+n^{\prime}}\right)
\end{aligned}
$$

Assumindo o valor de 0,1\% para a deformação de referência, os parâmetros $S_{e}$ e $E_{p}$ foram determinados para cada temperatura de ensaio, assim como os valores de $E$, K' e n' [7]. Estes valores estão apresentados na Tabela 2. Os modelos de Ramberg-Osgood e de Plasticidade Linear são comparados com os resultados experimentais, Figura 6.

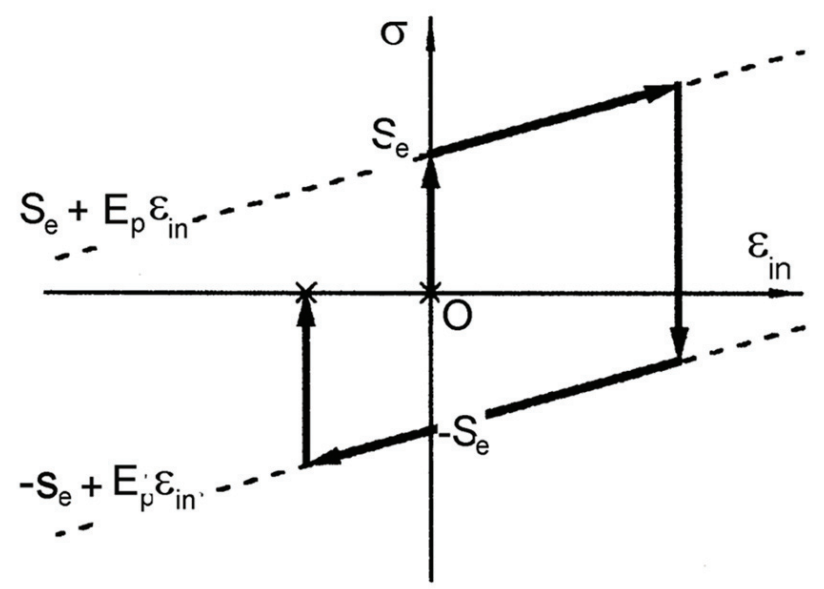

Figura 5. Representação esquemática do modelo plástico linear.
Pode ser observado que a relação de Ramberg-Osgood representa muito bem os resultados experimentais e que o modelo de Plasticidade Linear também fornece uma boa estimativa para baixas amplitudes de deformações inelásticas aplicadas.

As histereses de fadiga termomecânica podem ser determinadas tomando-se incrementos da deformação mecânica aplicada e calculando-se a tensão para cada intervalo, desde que um comportamento isotérmico seja assumido. É importante salientar que modelo proposto não leva em consideração a sensibilidade à taxa de deformação do material. Todas as constantes foram determinadas para uma taxa de deformação mecânica fixa, de $2 \times 10^{-4} \mathrm{~s}^{-1}$, que é a mesma usada nos ensaios de fadiga termomecânica.

As curvas obtidas a partir do modelo de Plasticidade Linear são comparadas com os dados das histereses de FTM experimentais apresentados na Figura 7, para um intervalo de deformação mecânica aplicada de I,4\%. Ainda, pode ser verificado que o modelo representa muito bem o comportamento do material em carregamentos em fase (EF) e fora de fase (FF), mas superestima as tensões e subestima os intervalos de deformação inelástica para o ciclo bi-térmico (BT).

A maior razão para o grande erro observado na simulação da FTM bi-térmica é a inabilidade do modelo proposto para tratar a deformação por fluência, que ocorre durante a manutenção da deformação mecânica, quando a temperatura varia de $600^{\circ} \mathrm{C}$ a $1.000^{\circ} \mathrm{C}$ em compressão e de $1.000^{\circ} \mathrm{C}$ a $600^{\circ} \mathrm{C}$, em tração. Este fato pode ser confirmado na Figura 8, que compara a curva

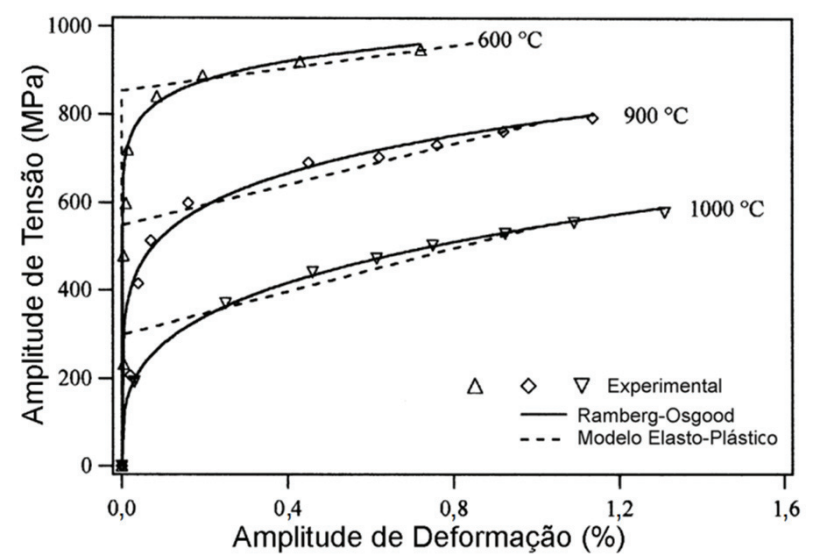

Figura 6. Comparação entre os modelos de Ramberg-Osgood e de plasticidade linear.

Tabela 2. Valores usados nos modelos de Ramberg-Osgood e de plasticidade linear

\begin{tabular}{cccccc}
\hline $\boldsymbol{T}\left({ }^{\circ} \mathbf{C}\right)$ & $\mathbf{E}(\mathbf{G P a})$ & $\boldsymbol{K}^{\prime}(\mathbf{M P a})$ & $\boldsymbol{n}^{\prime}$ & $\boldsymbol{S}_{\mathrm{e}}(\mathbf{M P a})$ & $\boldsymbol{E}_{\mathrm{p}}(\mathbf{G P a})$ \\
\hline $\mathbf{6 0 0}$ & 112 & 1.603 & 0,071 & 853 & 130 \\
$\mathbf{7 0 0}$ & 106 & 2.009 & 0,093 & 876 & 180 \\
$\mathbf{8 0 0}$ & 100 & 2.329 & 0,114 & 841 & 217 \\
$\mathbf{9 0 0}$ & 92,3 & 2.603 & 0,174 & 548 & 232 \\
$\mathbf{1 . 0 0 0}$ & 83,5 & 4.124 & 0,293 & 298 & 247 \\
\hline
\end{tabular}




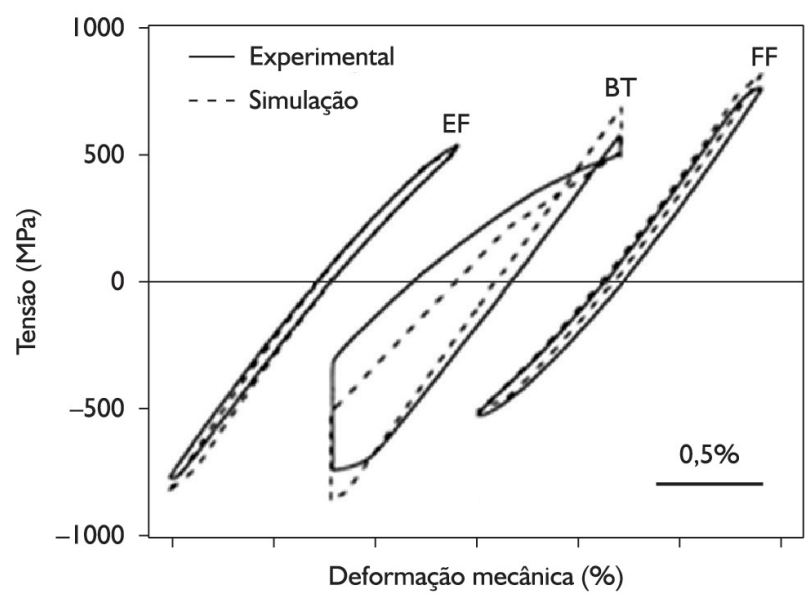

Figura 7. Comparação das curvas de histerese de FTM experimentais e calculadas.

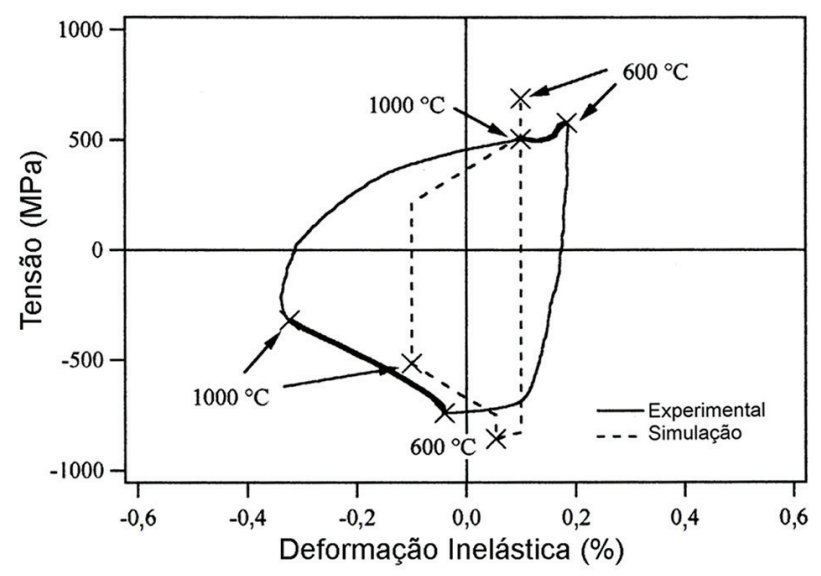

Figura 8. Análise da curva de histerese tensão-deformação inelástica, para o ensaio de FTM-BT.

de histerese inelástica experimental obtida para o ciclo de FTM-BT com a histerese calculada pelo modelo. As regiões onde ocorrem deformações inelásticas, durante a manutenção da deformação mecânica, são identificadas com linhas mais grossas, na curva de histerese experimental.
Pode ser notado que, embora o modelo subestime as deformações inelásticas reais, o formato geral da histerese pode ser razoavelmente previsto. Estes resultados indicam que é fundamental a utilização de um modelo de Plasticidade Linear para uma previsão mais acurada da resposta tensão-deformação desta liga, para temperaturas acima de $800^{\circ} \mathrm{C}$.

\section{CONCLUSÕES}

- A equação de Ramberg-Osgood ajusta-se muito bem o comportamento isotérmico experimental observado, para todo intervalo de temperatura estudado;

- O comportamento desta liga é fortemente afetado pela variação da temperatura e da taxa de deformação aplicada, especialmente no intervalo $800^{\circ} \mathrm{C}-1.000^{\circ} \mathrm{C}$;

- O modelo constitutivo Elástico-Linear-Plástico proposto foi capaz de reproduzir o comportamento observado para os ciclos de carregamento FTM mais comuns, em fase e fora de fase, para as condições investigadas neste trabalho;

- É necessário o desenvolvimento de um modelo constitutivo Elástico-Visco-Plástico para simular o comportamento do material estudado em condições gerais de carregamento a altas temperaturas, isto é, controle de tensão ou deformação e taxas de deformação variáveis.

\section{Agradecimentos}

Este trabalho foi financiado pela Fundação de Amparo à Pesquisa do Estado de São Paulo, Processo 99/0I540-0. Os autores agradecem ao Dr. M. Nazmy, da ABB, Suíça, pelo fornecimento do material e ao Professor A. Mortensen, Chefe do Laboratório de Metalurgia Mecânica do Instituto Federal de Tecnologia, Lausanne, Suíça, onde parte dos ensaios em altas temperaturas foi realizada.

\section{REFERÊNCIAS}

I Gell M, Duhl DN, Geamei AF. The development of single crystal superalloy turbine blades. In: High Temperature Alloys Committee of Metallurgical Society of AIME and the Power Activity of Materials Systems and Design Division of ASM. Proceedings of the Fourth International Symposium on Superalloys; 21 -25 Sep. 1980; Champion, USA. ASM; 1980. p. 205-2I4.

2 Harris K, Erickson GL, Schwer RE. Development of CMSX series of single-crystals alloys for advanced technology turbine components. In: American Institute of Mining, Metallurgical, and Petroleum Engineers. Proceedings of the TMS-AIME Fall Meeting; 1982; St. Louis, USA. Warrendale: TMS; 1982.

3 Date CG. TMF design considerations in turbine airfoil of advanced turbine engines. In: American Society of Mechanical Engineers. Proceedings of the Symposium, I 2 th ASME Winter Annual Meeting; I-6 Dec. 1991; Atlanta, USA. Nova lorque: ASME; 199I. p.59-64. 
4 Erickson GL, Harris K. DS and SX for industrial turbines. In: Coutsouradis D., et al., editors. Materials for advanced power engineering, Part II. Amsterdam: Kluwer Academic Publishers; 1994. p. I055-74.

5 American Society for Testing Materials. ASTM E606-04: Standard practice for strain-controlled fatigue testing. West Conshohocken: ASTM; 2004. http://dx.doi.org/I0.1520/E0606-04.

6 American Society for Testing Materials. ASTM E2368-04: Standard practice for strain controlled thermomechanical fatigue testing. West Conshohocken: ASTM; 2004. http://dx.doi.org/I0.1520/E2368-04.

7 Engler-Pinto CC Jr, Spinelli D. Cyclic behavior of the CM247LC-DS superalloy at high temperatures. In: Institut für Prozess-und Werkstofftechnik. Proceedings of Conferences on Applied Mechanics, Materials Science and Joining and Processes - CAMP; 3-4 Apr. 2002; Paderborn, Germany. Paderborn: Institut für Prozess-und Werkstofftechnik,Universität Paderborn; 2002. p. 2-I3.

Recebido: $29 / 10 / 2013$

Aceito: $25 / 03 / 2014$ 